\title{
Cirurgia bariátrica e depressão
}

\author{
Bariatric surgery and depression \\ Cirugía bariátrica y depresión
}

Gabrielle Souza Silveira Teles

ORCID: https://orcid.org/0000-0003-0560-4519

Universidade Tiradentes, Brasil

E-mail: gabriellessteles@gmail.com

Leda Maria Delmondes Freitas Trindade

ORCID: https://orcid.org/0000-0003-4300-4274

Universidade Tiradentes, Brasil

E-mail: leda.maria@ souunit.com.br

Renata Queiroz Corrêa

ORCID: https://orcid.org/0000-0003-4004-000X

Universidade Tiradentes, Brasil

E-mail: renata.queiroz10@gmail.com

Ana Clara Passos Melo

ORCID: https://orcid.org/0000-0003-0496-132X

Universidade Tiradentes, Brasil

E-mail: clarinhapassosm@gmail.com

Carolina Pinheiro Machado Teles

ORCID: https://orcid.org/0000-0002-6823-2540

Universidade Tiradentes, Brasil

E-mail: carolinapinheiroteles@ hotmail.com

Lucas Figueredo Moura

ORCID: https://orcid.org/0000-0001-5513-8398

Universidade Tiradentes, Brasil

E-mail: lucasdomicio123@gmail.com

\begin{abstract}
Resumo
A obesidade e a depressão são entidades clínicas cuja etiologia é a combinação de fatores genéticos, biológicos, ambientais e psicológicos capazes de comprometer a qualidade de vida. Objetivo: avaliar a prevalência da depressão antes e após a cirurgia bariátrica e identificar as características sociodemográficas e clínicas. Método: estudo transversal, retrospectivo, tipo survey realizado no período de 2020-2021. A amostra foi composta por 5.160 indivíduos de várias regiões do Brasil que realizaram cirurgia bariátrica. Utilizou-se questionário online Google Forms. Resultados: dos 5.160 participantes, 3199 (62\%) não tinham e não desenvolveram depressão após a cirurgia; $305(5,9 \%)$ tinham depressão e permaneceram após a intervenção; $1192(23,1 \%)$ tinham depressão e melhoraram no pós-cirúrgico e 464 (9\%) não tinham depressão e desenvolveram após a cirurgia. A idade média foi de 37-38 anos. Foi predominante: sexo feminino, cor de pele branca, ensino médio completo e indivíduos casados. A região sudeste foi a mais prevalente $(\mathrm{p}<0,001)$, assim como o etilismo antes e após a cirurgia. A prática de atividade física reduziu o número de casos de depressão após o procedimento. O acompanhamento psicológico foi realizado em $60 \%$ da amostra antes da cirurgia. O tempo de realização da bariátrica foi de 18-60 meses, sendo mais prevalente a técnica de Bypass Gástrico. A prevalência de outros transtornos psiquiátricos foi comum entre aqueles com histórico de depressão. Conclusão: a depressão foi prevalente antes e após a intervenção cirúrgica, entretanto a cirurgia bariátrica possibilitou a melhora do transtorno depressivo entre aqueles que já apresentavam a patologia antes do procedimento.
\end{abstract}

Palavras-chave: Cirurgia bariátrica; Depressão; Obesidade.

\begin{abstract}
Introduction: Obesity and depression are clinical entities whose etiology is a combination of genetic, biological, environmental and psychological factors capable of compromising quality of life. Objective: To evaluate the prevalence of depression before and after bariatric surgery and identify the sociodemographic and clinical characteristics. Methodology: cross-sectional, retrospective, survey-type study conducted in the period 2020-2021. The sample was composed of 5,160 individuals from various regions of Brazil who had undergone bariatric surgery. An online Google Forms questionnaire was used. Results: Of the 5,160 participants, 3199 (62\%) did not have and did not develop depression after surgery; 305 (5.9\%) had depression and remained after the intervention; 1192 (23.1\%) had depression and improved post-surgery and 464 (9\%) had no depression developed after surgery. The average age was 37-38 years. It was predominant: female gender, white skin color, complete high school and married individuals. The southeastern region was the most prevalent $(\mathrm{p}<0.001)$, as was alcoholism before and after surgery. Physical
\end{abstract}


activity reduced the number of cases of depression after the procedure. Psychological follow-up was performed in $60 \%$ of the sample before surgery. The realization time of bariatric surgery was 18-60 months, and the gastric bypass technique was most prevalent. The prevalence of other psychiatric disorders was common among those with a history of depression. Conclusion: Depression was prevalent before and after the surgical intervention, however, bariatric surgery allowed the improvement of depressive disorder among those who already presented the pathology before the procedure.

Keywords: Bariatric surgery; Depression; Obesity.

\section{Resumen}

Introducción: La obesidad y la depresión son entidades clínicas cuya etiología es una combinación de factores genéticos, biológicos, ambientales y psicológicos, capaces de comprometer la calidad de vida. Objetivo: evaluar la prevalencia de la depresión antes y después de la cirugía bariátrica e identificar las características sociodemográficas y clínicas. Metodología: Estudio transversal, retrospectivo, tipo encuesta, realizado en el periodo 2020-2021. La muestra estaba compuesta por 5.160 individuos de varias regiones de Brasil que se habían sometido a cirugía bariátrica. Se utilizó un cuestionario online de Google Forms. Resultados: De los 5.160 participantes, 3199 (62\%) tuvieron y no desarrollaron depresión después de la cirugía; 305 (5,9\%) tuvieron depresión y se mantuvieron; 1192 $(23,1 \%)$ tuvieron depresión y mejoraron después de la cirugía y 464 (9\%) no desarrollaron depresión. Predominaba el sexo femenino, el color de piel blanco, la educación secundaria completa y los individuos casados. La región del sureste era prevalente $(\mathrm{p}<0,001)$, así como el alcoholismo antes y después de la cirugía. La actividad física redujo el número de casos de depresión después del procedimiento. Seguimiento psicológico se realizó en el $60 \%$ de la muestra antes de la cirugía. El tiempo de cirugía bariátrica fue de 18 a 60 meses, y la técnica de bypass gástrico fue la más prevalente. La prevalencia de otros trastornos psiquiátricos era común entre quienes tenían antecedentes de depresión. Conclusión: La depresión era prevalente antes y después de la intervención quirúrgica, sin embargo, la cirugía bariátrica permitió la mejora del trastorno depresivo entre los que ya presentaban la patología antes del procedimiento.

Palabras clave: Cirugía bariátrica; Depresión; Obesidad.

\section{Introdução}

A obesidade é definida pela Organização Mundial de Saúde (2003) como o acúmulo anormal ou excessivo de gordura corporal em forma de tecido adiposo. É considerada doença de causa multifatorial, abrangendo fatores genéticos, comportamentais, metabólicos e ambientais (OMS, 2020). De acordo com a Vigilância de Fatores de Risco e Proteção para Doenças Crônicas por Inquérito Telefônico (2019), no Brasil, essa doença crônica aumentou 72\% nos últimos treze anos, saindo de 11,8\% em 2006 para 20,3\% em 2019. Segundo a Organização Mundial de Saúde (OMS), em 2025, a estimativa é de que 2,3 bilhões de adultos ao redor do mundo estejam acima do peso, sendo 700 milhões de indivíduos com obesidade, isto é, com um índice de massa corporal (IMC) acima de 30 (OMS, 2020).

O tratamento inicial recomendado para a obesidade envolve o acompanhamento nutricional, atividade física e uso de medidas farmacológicas (Fandiño et al., 2004; Segal \& Fandiño, 2002). A Diretriz Brasileira de Obesidade (2016) destaca que a cirurgia bariátrica se torna um recurso consistente nos casos de obesidade grave com falha documentada de tratamento clínico, proporcionando aos pacientes uma melhora de comorbidades clínicas e redução nos índices de mortalidade.

Segundo a Sociedade Brasileira de Cirurgia Bariátrica e Metabólica, o número de cirurgias aumentou 46,7\%, entre os anos 2012 e 2017, e o Brasil é o segundo país do mundo em número de cirurgias bariátricas. Este número só tende a crescer com a ampliação do rol de comorbidades para indicação de cirurgia bariátrica em pacientes com índice de massa corporal entre $35 \mathrm{~kg} / \mathrm{m}^{2}$ e 40kg/m², conforme Resolução n ${ }^{o}$ 2.131/2015, do Conselho Federal de Medicina (Nacional, [s.d.]). A cirurgia bariátrica, como um procedimento para o tratamento de obesos graves, tem se tornado cada vez mais popular, tornando evidente que os transtornos psiquiátricos são comuns entre esses pacientes no pré e no pós-operatório (Müller et al., 2019).

De acordo com a OMS, a depressão é um transtorno mental comum que afeta mais de 264 milhões de pessoas em todo o mundo e se caracteriza por tristeza persistente e falta de interesse ou prazer em atividades anteriormente gratificantes, podendo afetar também o sono, o apetite, cursar com fadiga e redução da concentração. A etiologia da depressão é complexa e multifatorial, envolvendo interações entre fatores sociais, psicológicos e biológicos (OMS, 2021). 
Dawes et al. (2016), em meta-análise, estimaram que 23\% dos pacientes submetidos à cirurgia bariátrica possuíam um transtorno de humor atual, mais comumente a depressão. A literatura sugere que a perda de peso após a cirurgia bariátrica está relacionada à redução nos níveis de depressão em curto e médio prazo (Zwaan et al., 2011; Mitchell et al., 2014). No entanto, nem todos os pacientes bariátricos experimentam ganhos de saúde mental com a intervenção cirúrgica que, em alguns casos, está relacionada à falta de perda de peso e às deformidades do contorno corporal devido ao excesso de pele (Kubik et al., 2013).

É importante compreender que diversos fatores biopsicossociais podem influenciar os resultados pós-cirúrgicos e que as preocupações pós-operatórias se modificam ao longo dos anos, à medida que os pacientes progridem através do período de perda de peso inicial em direção à estabilização ou, para alguns, a recuperação do peso (Mitchell et al., 2014). É necessário reconhecer que os pacientes precisam de acompanhamento multidisciplinar não apenas antes da cirurgia, mas também durante e após o procedimento, além da realização de estudos que abordem os problemas de saúde mental em pacientes que se submeteram a intervenção cirúrgica (Mendes et al., 2021; Müller et al., 2013). Dessa forma, este estudo teve como proposta avaliar a prevalência da depressão antes e após o procedimento cirúrgico, além de evidenciar a influência de características clínicas e sociodemográficas.

\section{Metodologia}

Estudo transversal, prospectivo e descritivo realizado no período de 2020 a 2021. Por se tratar de uma investigação no campo da saúde a amostragem foi por saturação, composta por indivíduos maiores de 18 anos que foram submetidos à cirurgia bariátrica, residentes em qualquer região do país. Os pacientes que realizaram cirurgia bariátrica e estavam presentes em grupos do Facebook voltados para a troca de experiência sobre a realização do procedimento foram convidados para participar da pesquisa através de questionário online disponibilizado na plataforma Google Forms. Os pesquisadores precisaram solicitar autorização dos moderadores dos grupos para que o questionário fosse divulgado entre os participantes. Além disso, os pesquisadores se mantiveram a disposição para esclarecer possíveis dúvidas e questionamentos através da própria rede social e/ou através de email.

Para ter acesso ao instrumento de pesquisa, os participantes entraram no link disponibilizado pelos pesquisadores e assinaram o Registro de Consentimento Livre Esclarecido - RCLE, elaborado de acordo com as diretrizes e normas regulamentadas de pesquisa envolvendo seres humanos atende à Resolução CNS n ${ }^{\circ}$ 510, de 07 de abril de 2016, do CNS/Ministério de Saúde - Brasília - DF. Os participantes não tiveram nenhum custo para participar da pesquisa e foram abordados uma única vez.

A amostra foi composta por 5.160 indivíduos, sendo excluídos da pesquisa os pacientes menores de 18 anos e os que realizaram mais de um tipo de cirurgia bariátrica. Para identificar os aspectos sociodemográficos e clínicos foi aplicado um questionário contendo as seguintes variáveis: idade, sexo, região do país, cor da pele, escolaridade, estado civil, uso de álcool, tabagismo, drogas ilícitas, prática de atividade física, acompanhamento com psicólogo e/ou psiquiatra, tempo de realização e tipo de cirurgia bariátrica, peso antes da cirurgia e peso atual e IMC anterior e atual. Outra variável contida no instrumento de pesquisa foi se obteve diagnóstico, antes ou depois da operação, por um médico psiquiatra de depressão, ansiedade, transtorno do pânico, compulsão alimentar e/ou fobias.

As variáveis categóricas foram descritas por meio de frequências absolutas e relativas percentuais. As variáveis contínuas foram descritas por meio de mediana e intervalo interquartil. A hipótese de independência entre variáveis categóricas foi testada por meio do teste Qui-quadrado de Pearson. A hipótese de aderência das variáveis contínuas à distribuição normal foi testada por meio do teste de Shapiro-Wilks. Uma vez que essa foi rejeitada, a hipótese de igualdade de medianas foi testada por meio do teste de Kruskal-Wallis. A hipótese de igualdade de duas medianas foi testada por meio do teste de Mann- 
Whitney-Bonferroni. O software utilizado foi o R Core Team 2021 (Versão 4.1.0). Estudo aprovado pelo CEP/UNIT/CAAE com parecer $n^{\circ} 4.371 .626$.

\section{Resultados}

Foram coletados os dados de 5.160 indivíduos que realizaram cirurgia bariátrica no período de novembro de 2020 a janeiro de 2021. De acordo com as respostas, os participantes foram divididos em grupos: grupo A: 3199 (62\%) não tinham e não desenvolveram depressão após a cirurgia; grupo B: 305 (5,9\%) tinham depressão e permaneceram com a comorbidade após a intervenção cirúrgica; grupo C: 1192 (23,1\%) tinham depressão e melhoraram após a cirurgia e o grupo D: 464 (9\%) não tinham depressão e desenvolveram após a cirurgia. Todos os grupos foram avaliados isoladamente e entre si (Tabela 1).

A média de idade dos indivíduos pesquisados foi de 37 anos, nos grupos A, C e D, e de 38 anos, no grupo B, sendo a mínima 32 e a máxima 45 anos. O sexo feminino foi o mais prevalente e estatisticamente significativo em todos os grupos. Dentre as regiões brasileiras, a região sudeste foi predominante entre os quatro grupos, principalmente entre o grupo A (59,9\%) e o grupo D (60,9\%). A cor de pele branca, o ensino médio completo e os casados foram predominantes em todos os grupos (Tabela 1).

Tabela 1: Dados sociodemográficos de pacientes submetidos à cirurgia bariátrica.

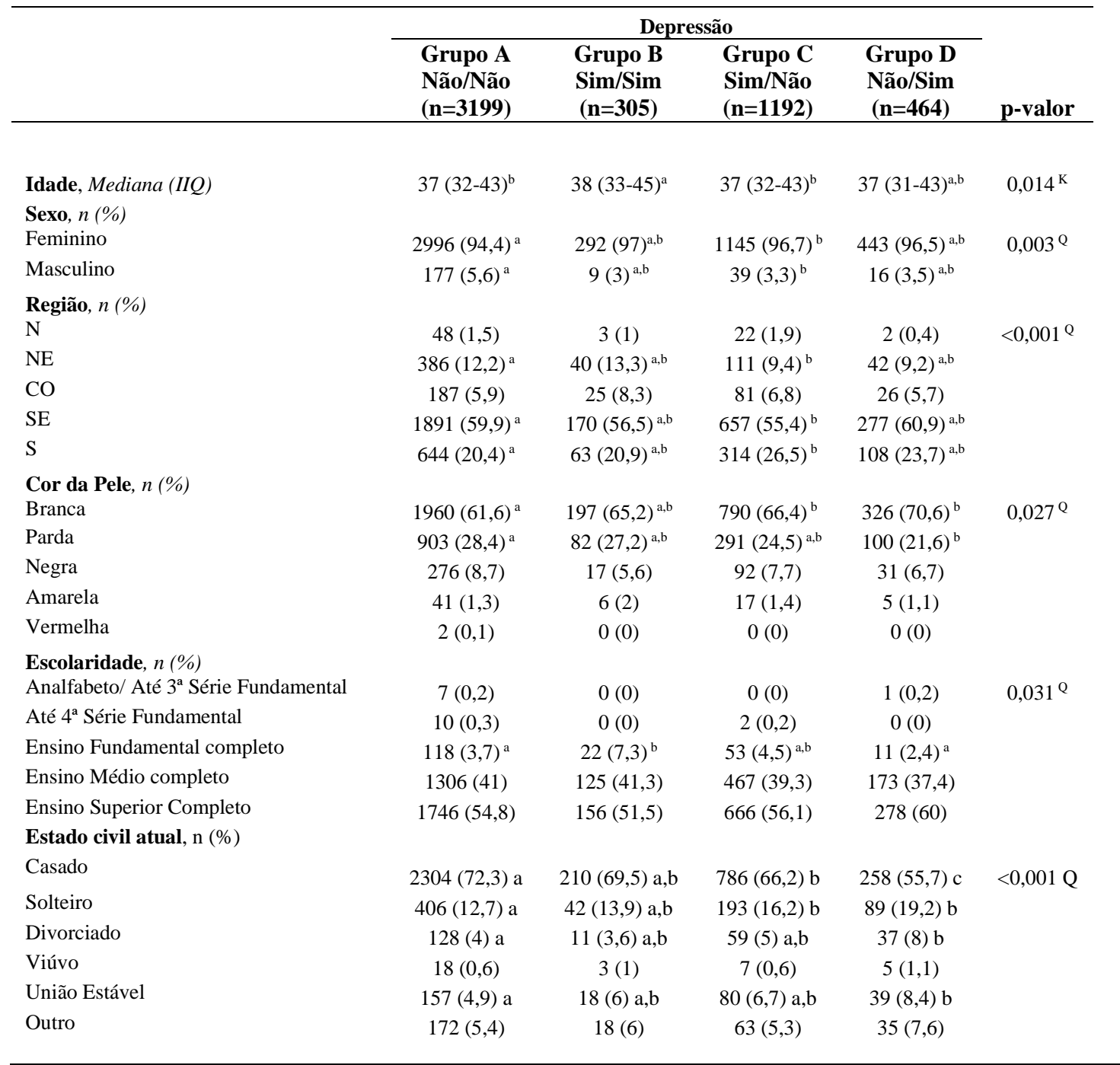

Legenda: $\mathrm{n}$ - frequência absoluta. \% - frequência relativa percentual. IIQ - Intervalo Interquartil. Q - Teste QuiQuadrado. K - Teste de Kruskal-Wallis. ${ }^{\text {a,b,c,d }}$ Subgrupos distintos ao nível de 5\% para o teste de Mann-WhitneyBonferroni. Fonte: Autores. 
Quando comparado os hábitos de vida entre os grupos, observou-se que a maioria nunca fez uso de cigarro e de drogas ilícitas. Em relação ao uso de bebidas alcoólicas, observou-se que em todos os grupos prevaleceu o uso antes e depois da cirurgia, sendo estatisticamente significativo entre os grupos D $(67,3 \%)$ e B $(62,4 \%)$. No que concerne à prática de atividades físicas, identificou-se que nos grupos $\mathrm{A}(31,7 \%$,$) e \mathrm{C}(31,3 \%)$ a maioria referiu prática antes e depois da cirurgia, enquanto que nos grupos B $(41,8 \%)$ e D $(34,5 \%)$ a maioria afirmou que nunca fez atividade física regularmente. Sobre acompanhamento com psicólogo e/ou psiquiatra, entre os grupos B, C e D a maior parte dos indivíduos referiram acompanhamento psicológico antes e depois da cirurgia, sendo estatisticamente mais significativo no grupo D (73,4\%,) (Tabela 2).

Tabela 2: Hábitos de vida e tipo de assistência psiquiátrica ou psicológica de pacientes submetidos à cirurgia bariátrica. Aracaju, 2020-2021.

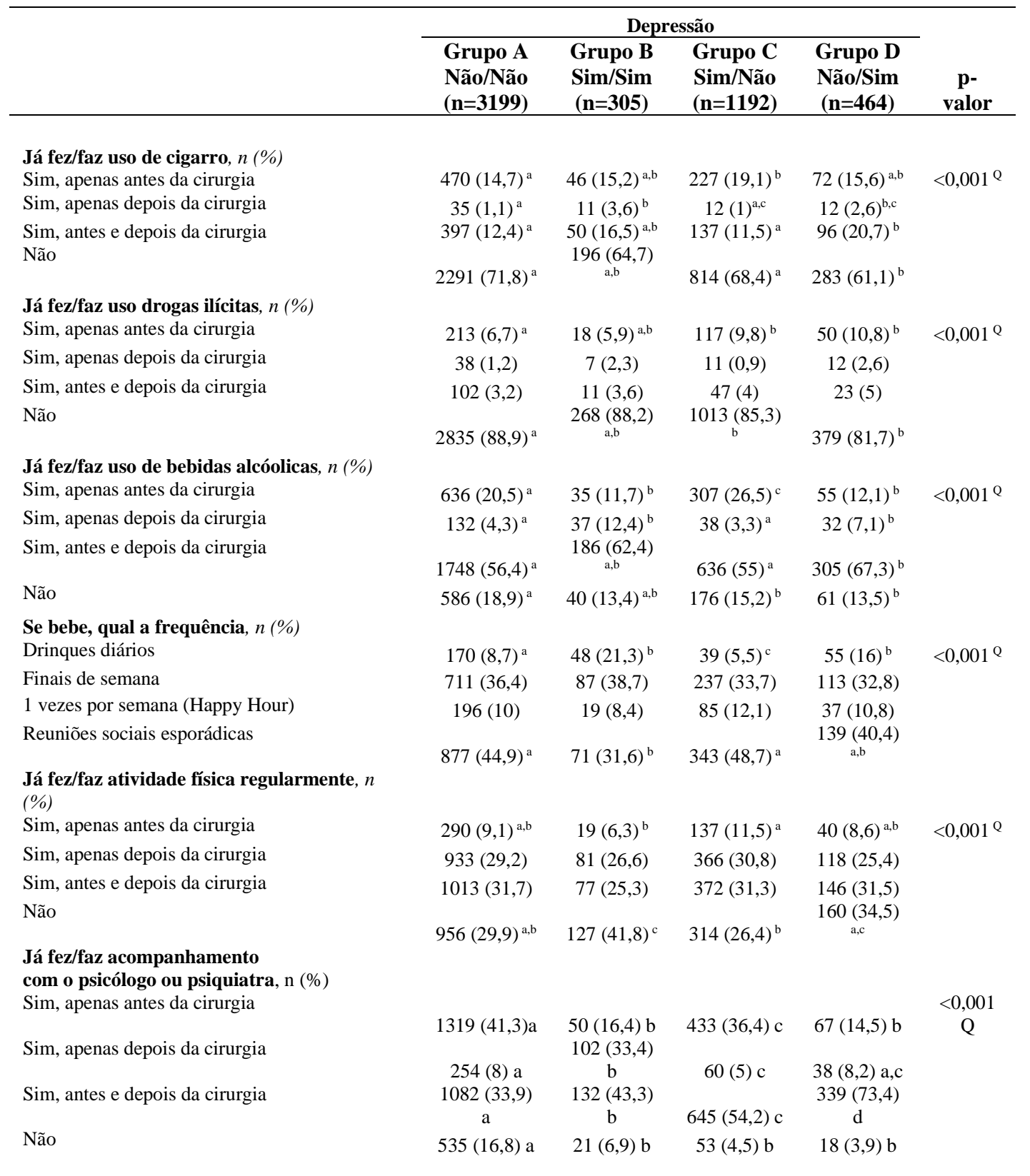

Legenda: $\mathrm{n}$ - frequência absoluta. \% - frequência relativa percentual. IIQ - Intervalo Interquartil. Q - Teste QuiQuadrado. K - Teste de Kruskal-Wallis. ${ }^{\text {a,b,c,d }}$ Subgrupos distintos ao nível de 5\% para o teste de Mann-WhitneyBonferroni. Fonte: Autores. 
Verificou-se que a mediana do tempo de realização da cirurgia bariátrica foi de 18 meses entre o grupo C, 24 meses no A, 36 meses no D e 48 meses no grupo B. Além disso, em todos os grupos, o tempo médio de realização da bariátrica foi entre 18 e 60 meses. Em relação ao tipo de cirurgia, houve grande predominância do Bypass Gástrico, principalmente no grupo B (86,6\%). As medianas dos pesos antes e depois da cirurgia foram, respectivamente, 113,7 quilogramas (kg) e $75 \mathrm{~kg}$ entre o grupo A, $117 \mathrm{~kg}$ e $75 \mathrm{~kg}$ entre o B, $112 \mathrm{~kg}$ e $75 \mathrm{~kg}$ entre o C e $115 \mathrm{~kg}$ e $74 \mathrm{~kg}$ entre o D. Sobre o IMC antes da cirurgia, a maioria dos pacientes eram obesos e, após a cirurgia, passaram a apresentar sobrepeso (Tabela 3).

Tabela 3: Dados clínicos dos pacientes submetidos à cirurgia bariátrica. Aracaju, 2020-2021.

\begin{tabular}{|c|c|c|c|c|c|}
\hline & \multicolumn{4}{|c|}{ Depressão } & \multirow[b]{2}{*}{$\begin{array}{c}\text { p- } \\
\text { valor }\end{array}$} \\
\hline & $\begin{array}{l}\text { Grupo A } \\
\text { Não/Não } \\
(n=3199)\end{array}$ & $\begin{array}{c}\text { Grupo B } \\
\text { Sim/Sim } \\
(n=305)\end{array}$ & $\begin{array}{l}\text { Grupo C } \\
\text { Sim/Não } \\
(n=1192)\end{array}$ & $\begin{array}{c}\text { Grupo D } \\
\text { Não/Sim } \\
(n=464) \\
\end{array}$ & \\
\hline $\begin{array}{l}\text { Há quanto tempo realizou a } \\
\text { cirurgia bariátrica, Mediana (IIQ) }\end{array}$ & $24(11-43)^{b}$ & $48(24,3-96)^{d}$ & $18(6-36)^{\mathrm{a}}$ & $36(23-60)^{\mathrm{c}}$ & $\begin{array}{c}<0,00 \\
1^{\mathrm{K}}\end{array}$ \\
\hline$n(\%)$ & & & & & \\
\hline Até 6 meses & $573(18,2)^{\mathrm{a}}$ & $5(1,7)^{\mathrm{b}}$ & $295(25,1)^{\mathrm{c}}$ & $30(6,6)^{d}$ & $<0,00$ \\
\hline 6-18 meses & $737(23,4)^{\mathrm{a}}$ & $27(9)^{b}$ & $327(27,8)^{\mathrm{c}}$ & $63(13,8)^{b}$ & \\
\hline $18-60$ meses & $1443(45,8)^{\mathrm{a}}$ & $151(50,3)^{a, b}$ & $457(38,9)^{\mathrm{c}}$ & $268(58,5)^{\mathrm{b}}$ & \\
\hline $60-120$ meses & $284(9)^{\mathrm{a}}$ & $69(23)^{b}$ & $69(5,9)^{\mathrm{c}}$ & $65(14,2)^{d}$ & \\
\hline$>120$ meses & $115(3,6)^{\mathrm{a}}$ & $48(16)^{b}$ & $27(2,3)^{\mathrm{a}}$ & $32(7)^{c}$ & \\
\hline \multicolumn{6}{|l|}{$\begin{array}{l}\text { Tipo de cirurgia bariátrica realizada, } \\
n(\%)\end{array}$} \\
\hline & $2544(80,1)^{\mathrm{a}}$ & $264(86,6)^{b}$ & $943(79,4)^{\mathrm{a}}$ & $373(80,7)^{\mathrm{a}, \mathrm{b}}$ & $\underset{Q}{0,023}$ \\
\hline Banda gástrica ajustável & $7(0,2)^{\mathrm{a}}$ & $4(1,3)^{\mathrm{b}}$ & $5(0,4)^{\mathrm{a}, \mathrm{b}}$ & $1(0,2)^{\mathrm{a}, \mathrm{b}}$ & \\
\hline Derivação bileopancreática & $1(0)$ & $1(0,3)$ & $1(0,1)$ & $0(0)$ & \\
\hline Gastrectomia vertical (sleeve) & $625(19,7)$ & $36(11,8)$ & $237(20)$ & $88(19)$ & \\
\hline Gastroplastia endoscópica & $1(0)$ & $0(0)$ & $1(0,1)$ & $0(0)$ & \\
\hline Peso antes da cirurgia, Mediana (IIQ) & $\begin{array}{l}113,7(104- \\
125)^{\mathrm{a}, \mathrm{b}}\end{array}$ & $\begin{array}{c}117(107- \\
130)^{\mathrm{c}}\end{array}$ & $\begin{array}{c}112(103- \\
123)^{\mathrm{a}}\end{array}$ & $\begin{array}{l}115(104,5- \\
127)^{b, c}\end{array}$ & $\begin{array}{c}<0,00 \\
1^{\mathrm{K}}\end{array}$ \\
\hline IMC antes da cirurgia, Mediana (IIQ) & $42,2(39,3-46)^{\mathrm{a}}$ & $\begin{array}{l}43,7(40,4- \\
47,6)^{\mathrm{b}}\end{array}$ & $\begin{array}{l}42,1(39,2- \\
45,9)^{\mathrm{a}}\end{array}$ & $\begin{array}{c}42,7(39,3- \\
46,8)^{\mathrm{a}, \mathrm{b}}\end{array}$ & $\begin{array}{c}<0,00 \\
1^{\mathrm{K}}\end{array}$ \\
\hline Peso atual, Mediana (IIQ) & $75(66-85,9)$ & $75(67-88)$ & $75(65-85)$ & $74(65-86)$ & $\underset{\mathrm{K}}{0,388}$ \\
\hline IMC atual, Mediana (IIQ) & $27,7(25-31,6)$ & $\begin{array}{l}27,7(25,1- \\
32,3)\end{array}$ & $28(24,8-31,6)$ & $\begin{array}{l}27,5(24,6- \\
31,9)\end{array}$ & $\underset{\mathrm{K}}{0,708}$ \\
\hline \multicolumn{6}{|l|}{ IMC antes da cirurgia, $n(\%)$} \\
\hline Sobrepeso & $1(0)$ & $0(0)$ & $1(0,1)$ & $0(0)$ & $\underset{Q}{1,000}$ \\
\hline Obesidade & $3164(100)$ & $301(100)$ & $1184(99,9)$ & $458(100)$ & \\
\hline $\begin{array}{l}\text { IMC atual, } n(\%) \\
\text { Abaixo do Peso }\end{array}$ & $3(0,1)$ & $1(0,3)$ & $3(0,3)$ & $0(0)$ & $\underset{\mathrm{Q}}{0,410}$ \\
\hline Peso Normal & $809(25,6)$ & $73(24,1)$ & $321(27,1)$ & $135(29,3)$ & \\
\hline Sobrepeso & $1290(40,8)$ & $117(38,6)$ & $459(38,7)$ & $170(36,9)$ & \\
\hline Obesidade & $1063(33,6)$ & $112(37)$ & $402(33,9)$ & $156(33,8)$ & \\
\hline
\end{tabular}

Legenda: $\mathrm{n}$ - frequência absoluta. \% - frequência relativa percentual. IIQ - Intervalo Interquartil. Q - Teste QuiQuadrado. K - Teste de Kruskal-Wallis. ${ }^{\text {a,b,c,d }}$ Subgrupos distintos ao nível de 5\% para o teste de Mann-WhitneyBonferroni. Fonte: Autores.

Correlacionando a depressão com outras patologias psiquiátricas observou-se que a incidência dos demais transtornos foi maior entre os pacientes que têm e/ou já tiveram depressão antes e/ou depois da cirurgia bariátrica (Tabela 4). 
Tabela 4: Associação da depressão com outras patologias mentais em pacientes submetidos à cirurgia bariátrica de acordo com cada grupo. Aracaju, 2020-2021.

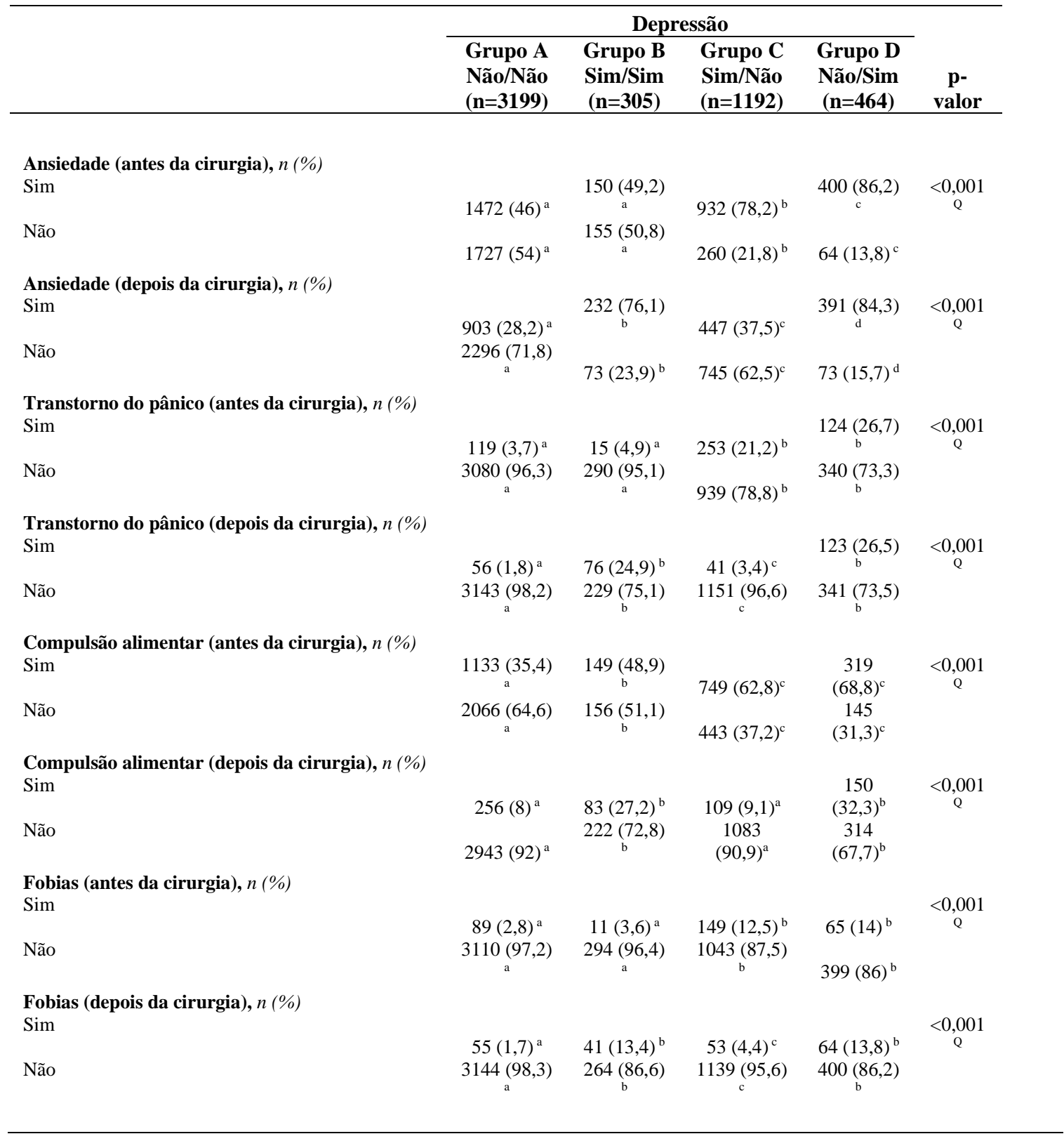

Legenda: $\mathrm{n}$ - frequência absoluta. \% - frequência relativa percentual. IIQ - Intervalo Interquartil. Q - Teste QuiQuadrado. K - Teste de Kruskal-Wallis. ${ }^{\text {a,b,c,d }}$ Subgrupos distintos ao nível de 5\% para o teste de Mann-WhitneyBonferroni. Fonte: Autores.

\section{Discussão}

Avaliando os 5.160 participantes por grupos foi possível identificar a relação entre a cirurgia bariátrica e o estado depressivo antes a após a intervenção e o quanto o procedimento cirúrgico teria ou não um papel significativo no comportamento emocional desses indivíduos. Mais de $60 \%$ dos participantes não tinham depressão e não desenvolveram após a intervenção, enquanto que $23,1 \%$ possuíam depressão e apresentaram melhora após a cirurgia. O grupo que não tinha depressão e desenvolveu após a cirurgia representou 9\% da amostra. Apenas 5,9\% faziam parte do grupo que tinha depressão e permaneceram com o transtorno após a cirurgia. 
Gill et al. (2019) avaliaram diversos estudos a respeito da associação da cirurgia bariátrica e a redução da depressão a partir de 24 meses de pós operatório. Através desses estudos foi observada alta prevalência de transtornos do humor no préoperatório dos bariátricos, principalmente da depressão. Essa condição também foi observada no grupo pesquisado, tendo sido representados pelos grupos B $(5,9 \%)$ e C $(21,3 \%)$ que já possuíam depressão antes de realizar o procedimento. O fato de possuírem depressão antes do procedimento parece ser um fator que reforça a manutenção do transtorno, quando se leva em consideração o tempo de realização do procedimento.

Em relação à presença de depressão no pós-operatório, observou-se que $9 \%$ dos pacientes desenvolveram a patologia após a cirurgia e $5,9 \%$ já tinham e permaneceram após o procedimento, enquanto a maioria $(23,1 \%)$ dos que tinham o transtorno depressivo apresentou melhora após a cirurgia. Um estudo de caso-controle, realizado no Reino Unido, observou que, no grupo cirúrgico, 20,3\% dos pacientes foram diagnosticados ou confirmados com depressão após a cirurgia, em comparação com 13,6\% do grupo não cirúrgico (Arhi et al., 2021). Esse dado difere dos resultados obtidos considerando que a incidência de depressão no pós-operatório foi menor do que no pré-operatório.

A faixa etária variou entre 32 a 45 anos, sendo a cor branca mais prevalente em todos os grupos. Foi mais comum o sexo feminino entre os participantes. De forma semelhante ao verificado, Guerra et al. (2020) observaram que a média de idade dos indivíduos que se submetem a bariátrica foi entre 18-45 anos e que o sexo feminino e a cor branca são de fato mais prevalentes.

Considerando a região brasileira que tinha maior registro de realização da cirurgia bariátrica, a região sudeste foi a mais prevalente entre os grupos abordados. Estudos que analisaram as características da cirurgia bariátrica realizada pelo sistema público de saúde do Brasil verificaram que a região sul foi responsável pela maioria das cirurgias, seguida pela região sudeste (Oliveira et al., 2021; Tonatto-Filho et al., 2019). Segundo dados do Instituto Brasileiro de Geografia e Estatística (2015), a população brasileira é basicamente formada por brancos e pardos e, na região sudeste, mais de 50\% da população se declara de cor branca, corroborando com os dados obtidos.

Em relação ao estado civil e a escolaridade, Bressan e Trevisol et al. (2019) observaram, em pesquisa com 71 pacientes submetidos a cirurgia bariátrica, o predomínio de indivíduos casados e com mais de 11 anos de estudo, semelhante à amostra pesquisada. No que concerne ao nível de instrução, observa-se que, segundo o IBGE (2016), a desigualdade ainda se revela com dados surpreendentes ao comparar as regiões do Brasil entre si. As regiões brasileiras sul e sudeste apresentam maiores índices de instrução, semelhante ao identificado na pesquisa em que o ensino superior completo e o ensino médio completo foram mais prevalentes na amostra estudada.

Quando avaliado os hábitos de vida, observou-se que a maioria dos participantes não fazia uso de cigarro e de drogas ilícitas antes e/ou depois da intervenção. Em relação ao uso de bebidas alcoólicas, houve prevalência do consumo antes e após a cirurgia nos quatro grupos. Bressan e Trevisol (2019) verificaram que 87,4\% dos participantes negaram tabagismo pré e póscirúrgico e $88,7 \%$ nunca fizeram uso de drogas ilícitas. Quanto ao etilismo, 84,5\% afirmou que nunca ingeriu álcool, diferindo deste estudo em que se mostrou predominante o uso pré e pós-cirúrgico.

No que se refere ao hábito de atividade física antes e após a cirurgia bariátrica, os dados revelaram que, o grupo dos participantes que tinham e permaneceram depressivos e os que não tinham e desenvolveram a depressão após o procedimento, não praticavam atividade física antes e nem após a cirurgia. Não foi possível comparar os resultados obtidos com outros estudos por falta de pesquisa que abordassem essa relação.

Sobre acompanhamento com psicólogo e/ou psiquiatra, entres os grupos B, C e D a maior parte dos indivíduos referiram acompanhamento psicológico antes e depois da cirurgia. Apenas no grupo A foi identificado que a maioria dos indivíduos realizava acompanhamento somente antes da cirurgia. Com o objetivo de identificar programas altamente conduzidos no contexto de terapia cognitivo-comportamental (TCC), Cheroutre et al. (2020) evidenciaram que o processo 
terapêutico foi realizado preferencialmente antes da cirurgia. Esse dado corrobora com a pesquisa, considerando que $60 \%$ do total da amostra (grupo A) realizaram acompanhamento psicológico/psiquiátrico apenas antes do procedimento. Destaca-se que esse grupo foi representado por aqueles que não tinham e não desenvolveram depressão após a intervenção cirúrgica.

O tempo médio de realização da bariátrica foi entre 18 e 60 meses, sendo a mediana do tempo de realização da cirurgia de 18 meses entre aqueles que tinham e permaneceram com depressão, 24 meses entre os que não tinham e não desenvolveram, 36 meses entre os que tinham e melhoraram e 48 meses entre os que não tinham e desenvolveram depressão. De forma semelhante ao presente estudo, a literatura mostra que o tempo de realização da cirurgia bariátrica interfere diretamente na presença da depressão.

White et al. (2015) relatou que 45\% da população do seu estudo apresentou escores de depressão clinicamente significativos imediatamente após a cirurgia. Essas pontuações significativas foram reduzidas para 13,3\% após 12 meses de acompanhamento e aumentaram para 17,5\% após 24 meses da cirurgia. Através de publicação, Dawes et al. (2016) demonstraram expressiva evidência quanto à redução da prevalência e da gravidade da depressão 12 meses após a cirurgia, mas os benefícios são menos claros quando avaliados depois de 24 meses de bariátrica. Outra pesquisa demonstrou que pacientes com depressão já diagnosticada tiveram uma redução no número de consultas relacionadas à depressão, em contraste com os pacientes sem a condição pré-existente que tiveram um risco aumentado de procurar atendimento médico por causa desse transtorno do humor (Arhi et al., 2021).

Quanto ao tipo de cirurgia, os dados evidenciaram predomínio do Bypass Gástrico. Semelhante ao verificado, Filho et al. (2019) observaram que o Bypass Gástrico foi responsável por 94,9\% dos procedimentos realizados no país quando comparado a outras técnicas cirúrgicas. Analisando o perfil clínico dos pacientes, constatou-se que antes da cirurgia a maioria dos pacientes apresentava IMC maior que 30, enquanto que no pós-operatório foi mais comum o IMC entre 24,9 e 29,9. A relação entre a alteração de peso e o transtorno depressivo foi uma variável não evidenciada na pesquisa, embora a literatura observe que existe uma associação entre mudança de peso e sintomas depressivos (Gill et al., 2019). Os estudos mostram que níveis elevados de citocinas pró-inflamatórias estão associados a uma série de subtipos da depressão, sendo a maioria notavelmente resistente ao tratamento (Strawbridge et al., 2015). Dessa forma, a perda de peso como resultado da bariátrica pode diminuir os níveis de citocinas pró-inflamatórias, melhorando os sintomas depressivos. No entanto, mais pesquisas são necessárias para investigar essa relação.

Correlacionando a depressão com outras patologias psiquiátricas, identificou-se que entre os pacientes diagnosticados com depressão há uma maior incidência dos demais transtornos (Gill et al., 2019). Analisando a amostra pesquisada observouse que antes da cirurgia existia uma elevada taxa de ansiedade em todos os grupos, mas, após a cirurgia, os níveis de ansiedade foram mais significativos apenas entre os pacientes que possuíam depressão. Rosik et al. (2005) observaram em estudo que os pacientes bariátricos pré-cirúrgicos frequentemente apresentam sintomatologias psiquiátricas e, pelo menos metade da amostra avaliada, poderia ser diagnosticada com no mínimo um transtorno psiquiátrico. Além disso, outra publicação também demonstrou que transtornos depressivos e outras doenças mentais foram mais prevalentes em pacientes cirúrgicos e, consequentemente, as taxas de eventos de automutilação deliberada também foram maiores nos bariátricos do que na população em geral (Morgan \& Ho, 2017).

Avaliando a compulsão alimentar observa-se que o número de pacientes diagnosticados com a patologia reduziu entre os quatro grupos, sendo essa redução menos expressiva entre os grupos que possuíam depressão após a cirurgia. Corroborando com o estudo, Machado et al. (2008) observaram que os pacientes portadores de obesidade mórbida submetidos à cirurgia bariátrica apresentavam indícios de compulsão alimentar antes e após a operação, relacionando a patologia com aspectos psicológicos como dificuldade em organizar emoções, ansiedade, depressão e estrutura emocional prejudicada. 
Entre os pacientes com depressão antes da cirurgia bariátrica foi observado que, analisando aqueles que melhoraram da patologia após o procedimento, houve uma redução do número de casos de transtorno do pânico e de fobias, enquanto que houve um aumento de ambos os transtornos entre aqueles que permaneceram com a depressão após a operação. De forma semelhante ao verificado analisando a relação entre a depressão e outros distúrbios psiquiátricos, pesquisas demonstram que a etiologia heterogênea dos transtornos mentais indica que é improvável que o efeito da cirurgia bariátrica seja homogêneo entre os diferentes grupos de patologias psiquiátricas. No entanto, apesar das diferenças, é possível reconhecer que há um aumento nas apresentações de serviços de saúde mental após a intervenção cirúrgica, principalmente entre aqueles que tiveram doenças psiquiátricas anteriores (Morgan et al., 2020).

\section{Conclusão}

A depressão, no grupo de pacientes bariátricos, foi prevalente tanto antes quanto após a intervenção cirúrgica. A cirurgia bariátrica possibilitou melhora do transtorno depressivo entre aqueles que já apresentavam a patologia antes do procedimento. Indivíduos com idade entre 37 e 38 anos, do sexo feminino, brancos, casados, com ensino superior completo e da região sudeste foram prevalentes neste estudo, assim como o consumo de álcool antes e após a cirurgia.

A prática de atividade física foi associada a um menor número de casos de depressão após o procedimento. Em relação ao acompanhamento psicológico/psiquiátrico, entre o grupo de pacientes que não tinha e não desenvolveu depressão após a intervenção cirúrgica foi mais comum o acompanhamento apenas antes do procedimento, destacando-se que esse grupo é responsável pela maior parte da amostra. Dessa forma, questiona-se se não houve o subdiagnóstico de depressão no pósoperatório entre esses participantes e, consequentemente, na amostra estudada.

Considerando o tempo de realização de cirurgia bariátrica, os pacientes que tinham a patologia e melhoraram no pósoperatório haviam realizado a cirurgia há menos tempo, enquanto que, os pacientes que não tinham a comorbidade e desenvolveram e os que tinham a patologia e permaneceram, haviam realizado o procedimento há mais tempo. Portanto, o benefício da cirurgia foi inversamente proporcional ao tempo de realização do procedimento.

Quanto à técnica cirúrgica, o Bypass Gástrico foi predominante na amostra. No que concerne à associação da depressão com outras patologias psiquiátricas, os pacientes com depressão após o procedimento tiveram maiores taxas de ansiedade, fobias e transtorno do pânico. Além disso, a redução do número de pacientes com compulsão alimentar após a cirurgia foi menos expressiva entres esses pacientes do que nos que tinham depressão e melhoraram após a intervenção.

Uma das limitações observadas nesta pesquisa foi a presença de uma amostra composta prioritariamente pelo sexo feminino, visto que os grupos em que o questionário foi divulgado era composto por mais mulheres do que homens. Outra limitação encontrada foi a impossibilidade de garantir que os indivíduos que responderam o questionário realizaram a cirurgia bariátrica, no entanto, os danos foram reduzidos aplicando esse questionário em grupos que tinham como objetivo a troca de experiências sobre o pós-operatório do procedimento.

\section{Referências}

Arhi, C. S., Dudley, R., Moussa, O., Ardissino, M., Scholtz, S., \& Purkayastha, S. (2021). The Complex Association Between Bariatric Surgery and Depression: A National Nested-Control Study. Obesity Surgery, 31(5), 1994-2001. https://doi.org/10.1007/s11695-020-05201-z

Bressan, J. de A., \& Trevisol, F. S. (2019). Avaliação da autoestima e depressão após cirurgia bariátrica. RBONE - Revista Brasileira de Obesidade, Nutrição e Emagrecimento, 13(79), 446-456.

Cheroutre, C., Guerrien, A., \& Rousseau, A. (2020). Contributing of Cognitive-Behavioral Therapy in the Context of Bariatric Surgery: A Review of the Literature. Obesity Surgery, 30(8), 3154-3166. https://doi.org/10.1007/s11695-020-04627-9

Dawes, A. J., Maggard-Gibbons, M., Maher, A. R., Booth, M. J., Miake-Lye, I., Beroes, J. M., \& Shekelle, P. G. (2016). Mental Health Conditions Among Patients Seeking and Undergoing Bariatric Surgery: A Meta-analysis. JAMA, 315(2), 150. https://doi.org/10.1001/jama.2015.18118 
Diretrizes Brasileiras de Obesidade 2016. ([s.d.]). Recuperado 28 de setembro de 2021, de https://abeso.org.br/wp-content/uploads/2019/12/DiretrizesDownload-Diretrizes-Brasileiras-de-Obesidade-2016.pdf

Fandiño, J., Benchimol, A. K., Coutinho, W. F., \& Appolinário, J. C. (2004). Cirurgia bariátrica: Aspectos clínico-cirúrgicos e psiquiátricos. Revista de Psiquiatria do Rio Grande do Sul, 26(1), 47-51. https://doi.org/10.1590/S0101-81082004000100007

Gill, H., Kang, S., Lee, Y., Rosenblat, J. D., Brietzke, E., Zuckerman, H., \& McIntyre, R. S. (2019). The long-term effect of bariatric surgery on depression and anxiety. Journal of Affective Disorders, 246, 886-894. https://doi.org/10.1016/j.jad.2018.12.113

Guerra, M. E., Jean, R. A., Chiu, A. S., \& Johnson, D. C. (2020). The effect of sociodemographic factors on outcomes and time to discharge after bariatric operations. The American Journal of Surgery, 219(4), 571-577. https://doi.org/10.1016/j.amjsurg.2020.02.046

Kubik, J. F., Gill, R. S., Laffin, M., \& Karmali, S. (2013). The Impact of Bariatric Surgery on Psychological Health. Journal of Obesity, 2013, 837989. https://doi.org/10.1155/2013/837989

Machado, C. E., Zilberstein, B., Cecconello, I., \& Monteiro, M. (2008). Compulsão alimentar antes e após a cirurgia bariátrica. ABCD. Arquivos Brasileiros de Cirurgia Digestiva (São Paulo), 21(4), 185-191. https://doi.org/10.1590/S0102-67202008000400007

Mendes, J. M. S., Neto, F. L., Queiroga, M. C., Lima, A. B. A. C. de, Abrantes, M. S. de A. P., \& Fonseca, E. N. R. da. (2021). Perfil psicopatológico dos indivíduos bariátricos tardio e sua associação com o reganho de peso. Research, Society and Development, 10(11), e521101119872-e521101119872. https://doi.org/10.33448/rsd-v10i11.19872

Mitchell, J. E., King, W. C., Chen, J.-Y., Devlin, M. J., Flum, D., Garcia, L., Pender, J. R., Kalarchian, M. A., Khandelwal, S., Marcus, M. D., Schrope, B., Strain, G., Wolfe, B., \& Yanovski, S. (2014). Course of Depressive Symptoms and Treatment in the Longitudinal Assessment of Bariatric Surgery (LABS-2) Study. Obesity (Silver Spring, Md.), 22(8), 1799-1806. https://doi.org/10.1002/oby.20738

Morgan, D. J. R., \& Ho, K. M. (2017). Incidence and Risk Factors for Deliberate Self-harm, Mental Illness, and Suicide Following Bariatric Surgery: A Statewide Population-based Linked-data Cohort Study. Annals of Surgery, 265(2), 244-252. https://doi.org/10.1097/SLA.0000000000001891

Morgan, D. J. R., Ho, K. M., \& Platell, C. (2020). Incidence and Determinants of Mental Health Service Use After Bariatric Surgery. JAMA Psychiatry, 77(1), 60-67. https://doi.org/10.1001/jamapsychiatry.2019.2741

Müller, A., Hase, C., Pommnitz, M., \& de Zwaan, M. (2019). Depression and Suicide After Bariatric Surgery. Current Psychiatry Reports, 21(9), 84. https://doi.org/10.1007/s11920-019-1069-1

Müller, A., Mitchell, J. E., Sondag, C., \& de Zwaan, M. (2013). Psychiatric Aspects of Bariatric Surgery. Current Psychiatry Reports, 15(10), 397. https://doi.org/10.1007/s11920-013-0397-9

Nacional, I. ([s.d.]). RESOLUÇÃO $N^{o}$ 2.131, DE 12 DE NOVEMBRO DE 2015-Imprensa Nacional. Recuperado 30 de setembro de 2021, de https://www.in.gov.br/materia

Número de cirurgias bariátricas no Brasil aumenta 46,7\%. (2018, julho 11). SBCBM. https://www.sbcbm.org.br/numero-de-cirurgias-bariatricas-no-brasilaumenta-467/

Oliveira, A. M. de, Costa, S. de S., Costa, I. S., \& Júnior, N. de J. P. B. (2021). Cirurgias bariátricas realizadas no Sistema Único de Saúde brasileiro entre 2010 e 2019. Research, Society and Development, 10(1), e47510111985-e47510111985. https://doi.org/10.33448/rsd-v10i1.11982

Organização Mundial da Saúde - OMS (2020). Obesity and overweight. http://www.who.int

Organização Mundial da Saúde - OMS (2021). Depression. https://www.who.int/health-topics/depression

PNAD Contínua 2016: $51 \%$ da população com 25 anos ou mais do Brasil possuíam no máximo o ensino fundamental completo | Agência de Notícias | IBGE. ([s.d.]). Recuperado 28 de setembro de 2021, de https://agenciadenoticias.ibge.gov.br/agencia-sala-de-imprensa/2013-agencia-de-noticias/releases/18992pnad-continua-2016-51-da-populacao-com-25-anos-ou-mais-do-brasil-possuiam-no-maximo-o-ensino-fundamental-completo

População brasileira é formada basicamente de brancos e pardos, diz IBGE. (2015, novembro 13). Agência Brasil. https://agenciabrasil.ebc.com.br/economia/noticia/2015-11/populacao-brasileira-e-formada-basicamente-de-brancos-e-pardos-diz-ibge

Rosik, C. H. (2005). Psychiatric symptoms among prospective bariatric surgery patients: Rates of prevalence and their relation to social desirability, pursuit of surgery, and follow-up attendance. Obesity Surgery, 15(5), 677-683. https://doi.org/10.1381/0960892053923815

Segal, A., \& Fandiño, J. (2002). Indicações e contra-indicações para realização das operações bariátricas. Brazilian Journal of Psychiatry, 24, 68-72. https://doi.org/10.1590/S1516-44462002000700015

Strawbridge, R., Arnone, D., Danese, A., Papadopoulos, A., Herane Vives, A., \& Cleare, A. J. (2015). Inflammation and clinical response to treatment in depression: A meta-analysis. European Neuropsychopharmacology, 25(10), 1532-1543. https://doi.org/10.1016/j.euroneuro.2015.06.007

Tonatto-Filho, A. J., Gallotti, F. M., Chedid, M. F., Grezzana-Filho, T. de J. M., \& Garcia, A. M. S. V. (2019). Bariatric Surgery In Brazilian Public Health System: The Good, The Bad And The Ugly, Or A Long Way To Go. Yellow Sign! ABCD. Arquivos Brasileiros de Cirurgia Digestiva (São Paulo), 32(4), e1470. https://doi.org/10.1590/0102-672020190001e1470

Vigitel_brasil_2019_vigilancia_fatores_risco. https://bvsms.saude.gov.br/bvs/publicacoes/vigitel_b rasil_2019_vigilancia_fatores_risco 
Research, Society and Development, v. 10, n. 13, e496101321573, 2021

(CC BY 4.0) | ISSN 2525-3409 | DOI: http://dx.doi.org/10.33448/rsd-v10i13.21573

White, M. A., Kalarchian, M. A., Levine, M. D., Masheb, R. M., Marcus, M. D., \& Grilo, C. M. (2015). Prognostic Significance of Depressive Symptoms on Weight Loss and Psychosocial Outcomes Following Gastric Bypass Surgery: A Prospective 24-Month Follow-Up Study. Obesity Surgery, 25(10), 1909-1916. https://doi.org/10.1007/s11695-015-1631-9

Zwaan, M., Enderle, J., Wagner, S., Mühlhans, B., Ditzen, B., Gefeller, O., Mitchell, J. E., \& Müller, A. (2011). Anxiety and depression in bariatric surgery patients: A prospective, follow-up study using structured clinical interviews. Journal of Affective Disorders, 133(1-2), 61-68. https://doi.org/10.1016/j.jad.2011.03.025 